\title{
ENFERMEdAdes CARdiovasCulares: Problema de SALUd EN LAS MUJERES
}

\author{
María José Cao Torija, María José Castro Alija y \\ Magdalena Santo Tomás Pérez \\ Universidad de Valladolid \\ mjcao@enf.uva.es
}

Recibido: 19-01-2010

Aceptado: $31-03-2010$

\section{Resumen}

Planteamos en el presente artículo una revisión de la incidencia, los principales factores de riesgo cardiovascular, y manifestaciones diferenciales de estas enfermedades entre mujeres y hombres por una parte desde el punto de vista biológico y por otra, y generalmente más olvidada desde el punto de vista del género, entendido como construcción social de lo femenino y lo masculino y poder así elaborar algunas conclusiones respecto a la prevención en general y específicamente en las mujeres.

Palabras clave: enfermedades cardiovasculares, mujeres, género.

\begin{abstract}
We propose in this article a review of the incidence, major cardiovascular risk factors, and differential signs of these diseases between women and men, on the one hand from the biological point of view and, on the other hand, and more generally neglected, from the point of view of the gender, understood as a social construction of the feminine and the masculine, and thus be able to draw some conclusions about prevention in general and specifically in women.
\end{abstract}

Key words: cardiovascular disease, women, gender. 


\section{Introducción}

A pesar del auge de las enfermedades tumorales o del renacer de algunas patologías infecciosas, las enfermedades cardiovasculares (ECV) se mantienen en los últimos años como la principal causa de muerte en todo el mundo, erigiéndose en una epidemia que no hace distinciones por sexo, edad, raza o localización geográfica. Aunque en el conjunto de la Unión Europea la tasa de mortalidad por enfermedades cardiovasculares disminuye, hay un número creciente de hombres y mujeres que conviven con una enfermedad cardiovascular. Las enfermedades cardiovasculares constituyen sin lugar a duda el principal problema de la población adulta de nuestro país y ocupan el primer lugar como causa de muerte para los mayores de 45 años.

Las enfermedades del corazón y del sistema circulatorio ocasionan 1,9 millones de muertes en la Unión Europea. Eso representa aproximadamente la mitad de todas las defunciones que se producen en los países europeos. El porcentaje es más elevado en las mujeres (55\% de todas las muertes) comparado con el de los hombres (43\% del total de fallecimientos). La mortalidad cardiovascular entre los hombres y mujeres de un estrato social bajo es incluso más elevada (Petersen, 2005). En 2007 se produjeron en España 385.361 defunciones, 13.883 más que en 2006. La principal causa de muerte fueron, una vez más, las enfermedades cardiovasculares (el 32,2\% del total) (Estadística de Defunciones Instituto Nacional de Estadística - INE).

La mala noticia es que las enfermedades del corazón son silenciosas y dan pocas señales de alerta. La buena noticia es que hoy día hay mucha mas información de la que había antes acerca de los pasos que se pueden tomar para mantener una salud cardiovascular óptima. 


\section{Clasificación de las enfermedades cardiovasculares (EECCVV) (ECV)}

Por enfermedad cardiovascular se entiende la enfermedad del corazón secundaria a la aterosclerosis que afecta las arterias coronarias que lo nutren y por extensión en especial la aterosclerosis de las arterias cerebrales, de la aorta y de las extremidades inferiores.

El término EECCVV es un concepto genérico que empleamos para referirnos a un conjunto de enfermedades diversas en sus causas o etiología y en sus manifestaciones clínicas que afectan al sistema cardiovascular. Según la versión X de la Clasificación Internacional de Enfermedades de la OMS (CIE-X Version, 2007) los grandes grupos de las enfermedades del aparato circulatorio son: Fiebre reumática aguda; Cardiopatías reumáticas crónicas; Enfermedades hipertensivas; Enfermedades cardíacas isquémicas; Enfermedad cardíaca pulmonar y enfermedades de la circulación pulmonar; Otras formas de cardiopatía; Enfermedades cerebrovasculares; Enfermedades de arterias, arteriolas y capilares; Enfermedades de vena, vasos linfáticos y nodos linfáticos, no clasificadas en otra parte; Otros trastornos del sistema circulatorio y trastornos sin especificar.

Cuando hablamos de enfermedades cardiovasculares, en particular en los países industrializados, nos estamos refiriendo mayoritariamente a las que en general comparten el mismo sustrato anatomopatológico, la lesión conocida como arteriosclerosis que afecta a las arterias y que sirve de base a varias enfermedades de importancia, como es la enfermedad arterial coronaria, la enfermedad cerebrovascular y algunas enfermedades de la aorta y circulación arterial periférica. 


\section{Epidemiología: Morbilidad y mortalidad desagregada por sexos}

En cuanto a mortalidad, España ocupa un lugar privilegiado entre los países industrializados en lo que respecta a cardiopatía isquémica, presentando unas tasas de mortalidad por esta enfermedad que se encuentran entre las más bajas del mundo industrializado. En enfermedad cerebrovascular presenta unas tasas intermedias entre este grupo de países.

La enfermedad cardiovascular (ECV) ha ido disminuyendo en España desde mediados de los años 70 hasta el momento actual, en parte debido, probablemente, al mejor control poblacional de un importante factor de riesgo de la enfermedad cerebrovascular como es la hipertensión arterial. La evolución de la cardiopatía isquémica es un poco diferente en el sentido de que prácticamente desde mediados de los años 70 las tasas han permanecido estables. Pese a esta tendencia favorable y pese a la posición relativa beneficiosa para España con respecto a otros países, las enfermedades cardiovasculares siguen siendo la principal causa de muerte en nuestro país, responsables del $32 \%$ de las muertes entre los hombres y del $44 \%$ de las muertes entre las mujeres (Muñiz Garcia, Crespo leiro y Castro Beiras, 2006).

En el año 2002 las ECV ocasionaron 125.797 muertes, lo que supone el 34\% de todas las defunciones (el 30\% en varones y el 39\% en mujeres). No obstante, por sexos, sólo en las mujeres la ECV es la primera causa de muerte (en los varones es la segunda, tras los tumores), y por grupos específicos de edad, las ECV son la primera causa de muerte sólo a partir de los 70 años de edad, situándose en segunda posición, detrás de los tumores, en personas de edades medias.

La enfermedad isquémica del corazón y la enfermedad cerebrovascular son las dos ECV que producen un mayor número de muertes cardiovasculares, con un $60 \%$ de la mortalidad cardiovascular total: el $31 \%$ por enfermedad coronaria (mayor en los varones, con un $39 \%$, que en las mujeres, con un 25\%) y el $29 \%$ por ictus (mayor en las mujeres, con un 31\%, que en los varones, con un 27\%) (INE, 2005) (fig.1). 
Figural: Porcentajes de enfermedades vasculares en varones y mujeres.

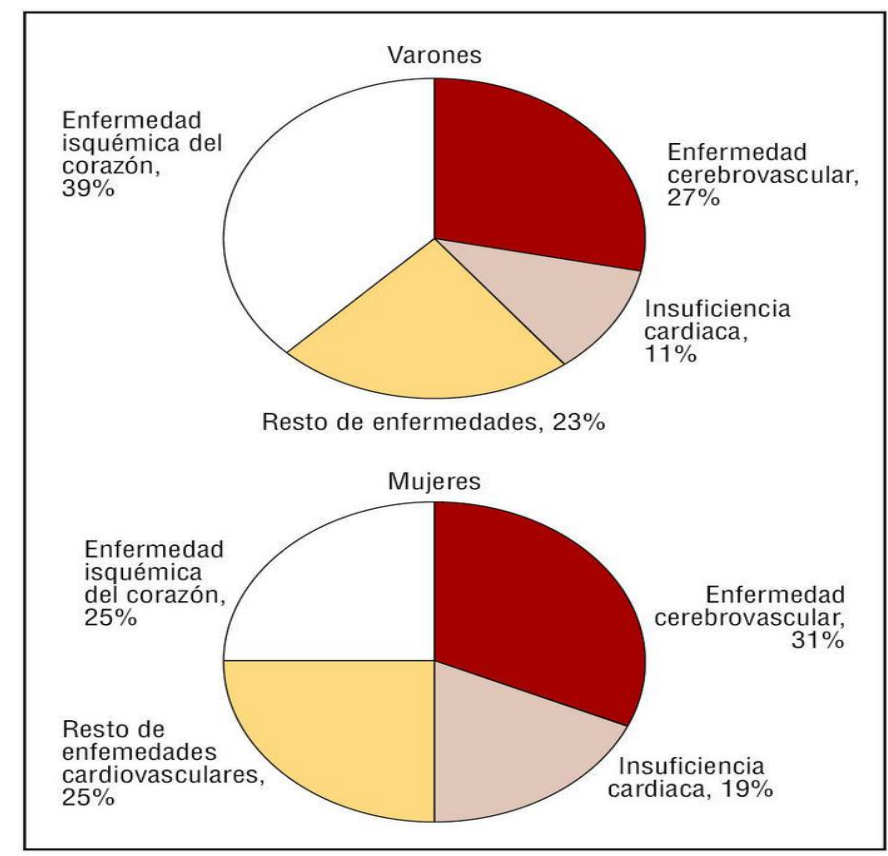

Fuente: Instituto nacional de Estadística 2005

La tercera ECV importante como causa de muerte es la insuficiencia cardiaca (IC), que ocasionó 18.571 muertes o el $15 \%$ de la mortalidad cardiovascular total, el $11 \%$ en varones y el $19 \%$ en mujeres, (INE, 2005) (fig.1). No obstante, la mortalidad registrada por IC puede infraestimar el verdadero impacto demográfico de esta causa de muerte, al ser la vía final común de muchas enfermedades que afectan al corazón.

Las tasas de mortalidad cardiovascular ajustadas por edad y las tasas específicas de edad son mayores en los varones (hasta los 85 años) (Villar Alvarez, 2005). Sin embargo, la mortalidad proporcional, las tasas brutas de mortalidad y el número absoluto de muertes son superiores en las mujeres. Esta aparente paradoja es el resultado de dos hechos bien conocidos: 
1. El mayor riesgo cardiovascular de los varones, reflejado en las tasas específicas de edad, de hecho, el riesgo coronario de los varones es similar al de las mujeres que son 10 años mayores que ellos.

2. La ECV es mucho más frecuente en las edades avanzadas de la vida, donde el número de mujeres es muy superior al de varones. Ello explica que las mujeres, a pesar de tener menos riesgo cardiovascular que los varones, presenten una mayor mortalidad proporcional bruta y un número más elevado de muertes por esta causa.

\section{En cuanto a la morbilidad}

La tasa de morbilidad hospitalaria de las ECV fue de 1.406 por 100.000 habitantes en el año 2002 en España, causando más de 5 millones de estancias hospitalarias. La tasa de morbilidad hospitalaria de la enfermedad isquémica del corazón fue de 365 por 100.000 habitantes (515 en los varones y 220 en las mujeres), y fue mucho más frecuente la presencia de otras formas de la enfermedad isquémica cardiaca (228 por 100.000 habitantes) que el infarto agudo de miocardio (IAM) (137 por 100.000 habitantes), a diferencia de lo que ocurre con la mortalidad. Respecto al ictus, la tasa de morbilidad fue de 266 por 100.000 habitantes (290 en los varones y 242 en las mujeres).

Por tanto, la morbilidad por enfermedad isquémica del corazón es casi el doble que la cerebrovascular en los varones, mientras que en las mujeres la morbilidad cerebrovascular es ligeramente superior a la isquémica. Por otro lado, la causa principal de hospitalización por ECV en ambos sexos corresponde al resto de enfermedades cardiovasculares, que en muchos de los casos cursan con IC. Este síndrome es la primera causa de hospitalización (no sólo por ECV) en los mayores de 65 años (INE, 2005). En España se pueden obtener datos sobre la incidencia de la cardiopatía isquémica del programa MONICA-Cataluña (Sans et al., 2001), del registro REGICOR y del estudio IBERICA (Fiol et al., 2001). Estos estudios indican que la incidencia del IAM, al igual que ocurre con la mortalidad, es más baja que en otros países 
desarrollados. Según estos estudios, la letalidad del IAM aumenta con la edad, y es mayor en el ámbito poblacional que en los pacientes hospitalarios. De hecho, muchas muertes por IAM se producen entre los pacientes que no llegan al hospital.

\section{Fisiopatología y factores de riesgo en mujeres y hombres}

La ateroesclerosis, como base de la ECV, es una alteración que empieza a desarrollarse en la juventud, mucho antes de que se manifieste clínicamente. Se caracteriza por la presencia de depósitos de lípidos en la capa íntima de las arterias, ya sea de grande, mediano o pequeño calibre produciendo con el tiempo, la denominada placa aterosclerosa que obstruye parcial o totalmente, la luz arterial, llevando a la isquemia miocárdica (Fig 2).

Figura 2: Arteria sana y arteria enferma

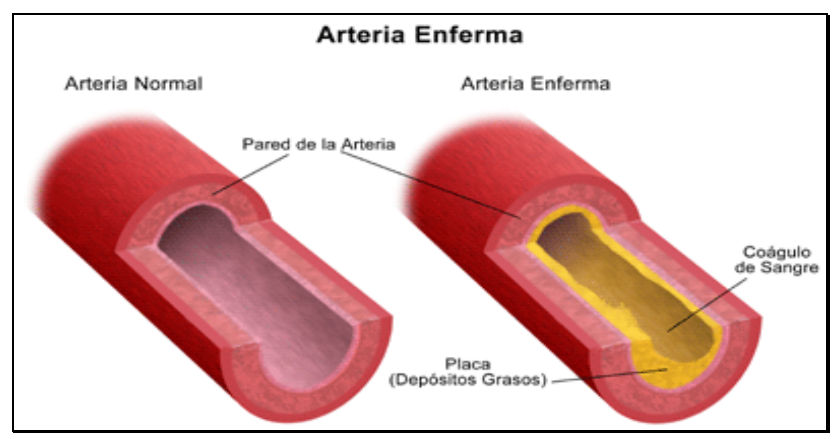

Fuente: Protege y restablece

Progresa lentamente con la edad si se está expuesto/a a determinados estilos de vida $\mathrm{y}$ factores de riesgo que desencadenan mecanismos y procesos celulares $\mathrm{y}$ bioquímicos altamente complejos formando el ateroma. 
Reacciones inflamatorias junto con factores mecánicos como la hipertensión, pueden llegar a ulcerar el ateroma. Si se produce esta ulceración, las plaquetas de la sangre acuden, se agregan y se produce la llamada trombosis, que puede obstruir total o parcialmente el lumen arterial e impedir la circulación de la sangre y por tanto el aporte de oxígeno necesario para los tejidos.

La consecuencia será la muerte celular o necrosis de los tejidos irrigados por la arteria ocluida, dando lugar así al infarto agudo de miocardio, (IM) al infarto cerebral o a la claudicación de las extremidades, en función de la arteria obstruida.

Existen múltiples diferencias en la anatomía y fisiologías cardiocirculatorias y cerebrales entre sexos, entre otras muchas, el corazón de la mujer es más pequeño que el del hombre. En la edad adulta pesa entre 300 y 350 g. en el hombre y entre 250 y $300 \mathrm{~g}$. en la mujer. La masa ventricular izquierda media en el hombre es de $177 \mathrm{~g}$. y de $118 \mathrm{~g}$. en la mujer. Las arterias coronarias de las mujeres son también de menor calibre.

Las arterias están recubiertas en su parte interna por una fina capa denominada endotelio. Las células de esta capa producen y captan multitud de moléculas, entre ellas las hormonas femeninas u estrógenos, por medio de receptores específicos. Varios de los mecanismos fisiopatológicos involucrados en los procesos de formación de la ateroesclerosis y de la trombosis y del tono arterial están modulados de forma diferencial por los estrógenos determinando la distinta frecuencia de las enfermedades ateroescleróticas en las mujeres, y su relación con las modificaciones hormonales ligadas a la menopausia.

Destacar por último que la presentación clínica en las mujeres pueden ser diferentes de los que presentan los hombres, las mujeres suelen sentir ardor en la región superior del abdomen y pueden tener mareo, malestar estomacal y sudoración, lo que podría dar lugar a no relacionar esta sintomatología con un problema cardiaco. 


\subsection{Factores de riesgo modificables e inmodificables}

Las causas de las ECV están bien definidas y son bien conocidas. Podemos distinguir:

\section{Factores de riesgo modificables}

Dieta inadecuada, inactividad física, aumento de la tensión arterial, de la glucemia, de los lípidos en sangre, sobrepeso y obesidad o consumo de tabaco.

\section{Factores de riesgo inmodificables}

La edad, el sexo y los antecedentes familiares no se pueden modificar. También hay una serie de determinantes subyacentes de ECV, como del resto de las enfermedades crónicas, es decir, "las causas de las causas", que son un reflejo de las principales fuerzas que rigen los cambios sociales, económicos y culturales: la globalización, la urbanización y el envejecimiento de la población.

\section{$\underline{\text { Edad }}$}

La gente de más edad tiene mayor probabilidad de desarrollar enfermedad coronaria que la gente joven. Afecta a los hombres tanto de mediana edad como mayores, pero en las mujeres es una enfermedad fundamentalmente de las ancianas (Vaccarino et al., 1995). En las personas mayores por los cambios anatómicos y fisiológicos que ocurren con el envejecimiento aparecen factores de riesgo, entre ellos la hipertensión. Otros factores de riesgo tal como fumar y el estilo de vida sedentario, son conductas de riesgo cuyos efectos pueden acumularse a lo largo de muchos años incrementando el riesgo en personas de edad. Por ejemplo: el peso corporal puede incrementarse a lo largo del tiempo y resultar en obesidad si un adulto de más edad no quiere seguir con un estilo de vida más activo y unas pautas de alimentación adecuadas. Las personas mayores tienen una combinación de factores de riesgo que pueden ser particularmente letales. 


\section{$\underline{\text { Sexo }}$}

El sexo es un factor de riesgo que tiene una estrecha relación con la edad, el estudio Framingham Study of Coronary Risk (1987) confirma este factor de riesgo. Existe una gran diferencia en la incidencia de cardiopatía isquémica en los varones y en las mujeres antes de los 50 años, se considera que, por debajo de esta edad, las mujeres tienen un riesgo diez veces menor que los varones de desarrollar ECV. Esta diferencia va disminuyendo a medida que avanza la edad, siendo la menopausia un punto de inflexión claro en el aumento de dicha incidencia en las mujeres, no obstante, no todos los autores están de acuerdo en esto; de cualquier modo, la menopausia produce numerosas alteraciones metabólicas que podrían poseer cierto riesgo aterogénico (Alegría, et al., 1991). En comparación con los hombres, las mujeres mayores, tienen más enfermedades concomitantes, los aspectos psicosociales son diferentes y tienen en general una menor capacidad física. Existen otros problemas que deben tenerse en cuenta como la conveniencia de la terapia hormonal sustitutiva, los efectos del embarazo sobre el corazón y la incidencia elevada de la hipertensión en las mujeres.

\section{Historia familiar}

El aspecto hereditario de los trastornos coronarios ha sido objeto de numerosos estudios, la influencia genética desempeña un papel importante, sobre todo, en los casos de aparición precoz de la enfermedad. No obstante, el riesgo de heredar la enfermedad varía ampliamente de unas familias a otras, dependiendo de la causa subyacente. Algunas influencias genéticas en el riesgo de enfermedad coronaria están mediadas vía reactividad, los resultados en gemelos y estudios familiares sugieren efectos genéticos significativos en responsividad cardiovascular al estrés (Garfinkel y Spano Ditto, 1992). 


\subsection{Factores de riesgo considerando el género}

\section{Tabaquismo y anticonceptivos orales (AO)}

El tabaquismo es un factor de riesgo de ECV, potencialmente modificable. Los riesgos asociados con él son sustancialmente más altos en mujeres que en hombres y no dependen de la edad. La frecuencia más alta de eventos cardíacos en hombres jóvenes en comparación con mujeres premenopáusicas se relacionaría con el papel protector de los estrógenos endógenos; la declinación rápida de los niveles de estrógenos luego de la menopausia explicaría los cambios desfavorables en el metabolismo de los lípidos y de los carbohidratos. Estas mismas modificaciones se observan en mujeres con insuficiencia ovárica prematura. Una mujer que fuma antagoniza el efecto beneficioso de sus estrógenos.

El tabaquismo en combinación con el uso de anticonceptivos orales puede ocasionar un incremento de los casos de IM en mujeres de más de 35 años. Sin embargo, el tabaquismo, independientemente del tratamiento con anticonceptivos, reviste una importancia particular por su asociación con la trombogénesis. Por el contrario, la utilización de anticonceptivos orales combinados no se asocia con aumento sustancial del riesgo de IM en mujeres sanas no fumadoras. No obstante, debe tenerse mucho cuidado cuando se indican AO a mujeres de más de 34 años que fuman. La anticoncepción oral también se acompaña de aumento del riesgo de enfermedad vascular isquémica, especialmente en mujeres que fuman mucho (Vessey, Painter y Yeates, 2003).

\section{$\underline{\text { Alcohol }}$}

El riesgo de cardiopatía isquémica en las personas que beben cantidades moderadas de alcohol es menor que el de los que no beben alcohol. Se considera una cantidad moderada de alcohol, una ración $(120 \mathrm{cc})$ de una bebida alcohólica fermentada de baja graduación (vino, cereza) al día para las mujeres y dos al día para los hombres. 
Las mujeres metabolizan el alcohol más lentamente que los hombres, por una menor producción de alcohol deshidrogenasa y por su menor superficie corporal en general. El alcohol en exceso puede elevar los triglicéridos, más en mujeres, aumenta la presión arterial y producir arritmias, insuficiencia cardiaca e ictus. Así mismo contribuye a la obesidad por un aporte adicional de calorías (Stuardo y Navarro, 2003).

\section{Dislipemia}

Conjunto de patologías caracterizadas por alteraciones en la concentración de lípidos sanguíneos en niveles que involucran un riesgo para la salud. Comprende situaciones clínicas en que existen concentraciones anormales de colesterol total (CT), colesterol de alta densidad (C-HDL), colesterol de baja densidad (C-LDL) y/o triglicéridos (TG). Las dislipidemias constituyen un factor de riesgo mayor y modificable de enfermedad cardiovascular, en especial coronaria. El colesterol transportado en lipoproteínas de baja densidad (C-LDL) está directamente correlacionado con el riesgo de enfermedad coronaria. El colesterol que forma parte de lipoproteínas de alta densidad (C-HDL) está inversamente correlacionado con el riesgo coronario. Las lipoproteínas de muy baja densidad (VLDL), contienen la mayoría de los TG del suero y algunas de sus formas son igualmente aterogénicas. La concentración de colesterol es un factor de riesgo significativo de ECV en hombres y en mujeres; el riesgo es bastante parecido en sujetos de los dos sexos y se eleva con la edad. El riesgo conferido por la hipertrigliceridemia es sustancialmente mayor en mujeres y desciende con la edad (ASOCIMED - guías clínicas, 2001).

\section{Diabetes}

La frecuencia aumenta de manera considerable con la edad y es mayor en mujeres de edad avanzada en comparación con sus pares masculinos. Los niveles altos de testosterona en mujeres aumentan la probabilidad de diabetes; las mujeres con antecedente de diabetes gestacional también tienen riesgo considerablemente mayor de presentar este trastorno en el futuro. La diabetes representa un factor importante de 
riesgo cardiovascular y se comprobó que las mujeres diabéticas tienen más probabilidad de presentar IM en comparación con otras pacientes con síndromes coronarios agudos; además, la mortalidad intrahospitalaria es más alta. El riesgo relativo de muerte por ECV y de IM no fatal atribuible a la diabetes también es superior en mujeres (Collins, et al., 2007).

\section{$\underline{\text { Sobrepeso y obesidad }}$}

El riesgo de muerte por ECV aumenta en relación con el índice de masa corporal. El riesgo relativo ajustado a la edad de mortalidad por ECV está aumentado y estudios epidemiológicos a largo plazo han mostrado que la mortalidad coronaria aumenta entre un $4 \%$ y un $6 \%$ de media por cada unidad adicional de IMC, comenzando por uno de 20 a 24 (Conroy et al., 1996).

La obesidad, y fundamentalmente la obesidad central, son más comunes en mujeres que en hombres. El aumento leve del peso, independientemente de la actividad física, confiere mayor riesgo de muerte en mujeres. En las sociedades occidentales, el peso corporal aumenta con la edad en ambos sexos, pero el ritmo de aumento en la mujer es más acelerado que en los hombres de la misma edad. Dado que este rápido aumento de peso acostumbra a depositarse mayoritariamente en el abdomen, éste es el principal factor contribuyente al deterioro del perfil de riesgo cardiovascular en las mujeres post-menopaúsicas puesto que contribuye en gran manera a la elevación de la presión arterial, colesterol y niveles de glucemia. Si los lípidos intraabdominales son muy abundantes son causantes del síndrome metabólico y la resistencia a la insulina. Según la Federación Internacional de Diabetes (Rolla, 2003) existe obesidad abdominal cuando el perímetro de una persona (medido por encima de las crestas iliacas y a nivel del ombligo) es mayor a 94 centímetros en los varones y 80 centímetros en las mujeres europeas respectivamente.

En el denominado estudio IDEA (Día Internacional de Evaluación de la Obesidad Abdominal) (Haffner, 2007), los resultados fueron contundentes. Comprobaron que en todas las regiones estudiadas existe una fuerte relación entre el 
perímetro de la cintura y las enfermedades cardiovasculares, tanto en las poblaciones más obesas como en las más delgadas, es decir, que el riesgo de enfermedades cardiovasculares aumenta con el tamaño de la circunferencia abdominal, independientemente del índice de masa corporal ó IMC. Además, el estudio IDEA demostró que a mayor nivel educativo, menor IMC y que entre personas desempleadas y jubiladas ese índice es más alto que en los trabajadores activos.

\section{Hipertensión arterial}

La presión arterial aumenta con la edad en ambos sexos. Si bien las mujeres tienen niveles inferiores a las de los hombres antes de la menopausia, las cifras se elevan mucho más después de ésta, y a partir de los 55 años superan a las de los hombres. La presión arterial alta aumenta el esfuerzo del corazón, acelera el proceso de endurecimiento de las arterias y aumenta el riesgo de sufrir un infarto agudo de miocardio, un ictus y una insuficiencia cardíaca.

Cuando la hipertensión coexiste con otros factores de riesgo, la probabilidad de infarto o ictus aumentan muchas veces. Las mujeres que tienen antecedentes familiares de hipertensión arterial y las mujeres con sobrepeso también tienen un mayor riesgo. Es el principal factor de riesgo de padecer insuficiencia cardiaca o ictus.

Cada aumento de $20 \mathrm{mmHg}$ en la presión arterial sistólica, o de $10 \mathrm{mmHg}$ en la presión arterial diastólica, dobla el riesgo de enfermedad cardiovascular, en todo el intervalo desde 115/75 hasta $185 / 115 \mathrm{mmHg}$. Además, como hemos visto anteriormente, la hipertensión raramente existe aislada, sino que habitualmente coexiste con otros factores de riesgo y así el riesgo de sufrir enfermedad cardiovascular aumenta exponencialmente, de forma que el efecto final de la combinación de varios factores es mayor que la suma de los factores individuales (SEMERGEN, 2005).

\section{$\underline{\text { Síndrome Metabólico (SM) }}$}

Es la agrupación de 3 o más factores de riesgo cardiovascular, entre ellos: obesidad central, alteración en el metabolismo de la glucosa, hipertrigliceridemia, 
reducción del HDLc e hipertensión. El síndrome metabólico eleva la probabilidad de aparición de diabetes, ECV y mortalidad cardiovascular. Un amplio estudio epidemiológico (Collins et al., 2007) mostró que el 56\% y 72\% de las mujeres presentan síndrome metabólico, en comparación con el $40 \%$ y $59 \%$ de los hombres al aplicar los criterios de definición del National Cholesterol Education Program Adult Treatment Panel III (NCEP-ATP III) y de la International Diabetes Federation, respectivamente. Se considera un elemento importante en la epidemia actual de diabetes y de ECV, de manera que se ha convertido en un problema de salud pública importante en todo el mundo (Eckel, Grundy y Zimmet, 2005).

\section{$\underline{\text { Sedentarismo }}$}

Los efectos del ejercicio sobre los factores de riesgo coronarios son multifactoriales y es probable que el proceso arteriosclerótico quede influido por medio de un efecto positivo sobre ciertos factores de riesgo coronarios, así como mediante otros mecanismos más directos. Científicamente definimos sedentarismo como el estado que se caracteriza por la falta de actividad física mínima diaria recomendada para producir los fenómenos adaptativos que disminuyan el riesgo cardiovascular o de enfermedades relacionadas con esa falta de actividad física. Kahn et al. (2002) llevaron a cabo un estudio de 54 investigaciones sobre el sedentarismo y determinó que las personas sedentarias tenían un riesgo dos veces mayor de presentar ECV, siendo esta su conclusión más relevante. Lo que llevó a la Asociación Americana del Corazón (American Heart Association) a declarar la inactividad física como un factor de riego para el desarrollo de ECV. El efecto, puede deberse en parte a la modificación apropiada de los lípidos sanguíneos, la presión arterial, los factores de la coagulación y el estrés psicológico.

El sedentarismo es muy común en las mujeres de edad intermedia y representa un factor de riesgo sustancial de ECV. Respecto al grado de beneficio obtenido con el ejercicio, cualquier ejercicio parece ser mejor que ninguno; pero el ejercicio de intensidad alta parece tener ciertos efectos beneficiosos que no se observan en ejercicios 
de intensidad baja. En general la mejor forma de ejercicio es caminar como mínimo 10 ó 20 minutos al día, tal hecho tiene un efecto mesurable sobre la salud cardiovascular.

\section{Menopausia}

Se estima que las mujeres viven más que los hombres; para el año 2050 se considera que más del $30 \%$ de las mujeres tendrá 60 años o más y, en consecuencia, aumentará el número de mujeres en la transición menopáusica. En la medida que se incrementa la expectativa de vida ( $\sin$ cambios en la edad de la menopausia), en un futuro cercano las mujeres pasarán entre el 30\% y $40 \%$ de sus vidas en estado posmenopáusico. En este período, las mujeres deben ser muy bien evaluadas para detectar y corregir los factores de riesgo cardiovascular. Sin embargo, se suele prestar mucha menos atención a la prevención. El control de la presión arterial, de la dislipidemia, los trastornos del metabolismo de la glucosa y el tabaquismo son algunos de los factores de riesgo, potencialmente modificables.

\subsection{Factores psicosociales}

La investigación sistemática de la relación entre el corazón y la mente empezó a fines de los años 50 con el trabajo pionero de Meyer Friedman y Ray Rosenman, (Meyer y Rosenman 1974) dos cardiólogos de San Francisco que acuñaron el término Patrón de Comportamiento TIPO A (PCTA). Desde esa fecha se ha generado un gran monto de investigación entre los factores psicosociales y la enfermedad cardiovascular (ECV). Los factores estudiados que aparecen como los más importantes son: 


\section{PCTA}

Es un fenómeno clínico complejo caracterizado por una constelación de conductas que incluyen la competitividad, ira, hostilidad libremente flotante y bien racionalizada, urgencia de tiempo, impaciencia, discurso vigoroso, y un número de signos psicomotores. Se observa en una persona involucrada agresivamente en una lucha crónica e incesante para lograr más y más en el menor tiempo posible,. Los procesos fisiopatológicos que acompañan la lucha crónica del PCTA, aceleran la aterogénesis conducente a una prematura enfermedad coronaria.

El PCTA puede ejercer su papel en la etiología y curso de la enfermedad, bien actuando directamente en el proceso aterogénico, bien funcionando como detonante que precipite eventos clínicos en pacientes con una aterosclerosis coronaria ya desarrollada (Meyer y Rosenman 1974). Debemos apuntar el inadecuado tamaño y composición de las muestras estudiadas en algunas investigaciones al respecto, puesto que, aunque se hayan realizado estudios con muestras suficientemente representativas, gran parte de los estudios se han realizado con muestras compuestas casi exclusivamente por individuos de sexo masculino.

\section{Ira y Hostilidad}

En 1987 R. B. Williams acuño el término complejo de hostilidad para describir una orientación cínica, escéptica, sarcástica, despreciativa, desconfiada y pesimista en la interacción interpersonal y vida en general. Y pensó que podía estar más fuertemente relacionada con la enfermedad coronaria que el PCTA global.

Los sujetos hostiles tienen mayor probabilidad de padecer episodios de ira, los cuales si no son racionalizados y canalizados adecuadamente, pueden ser reprimidos por el sujeto o pueden desembocar en conductas agresivas, aumentando así el riesgo de llegar a sufrir un trastorno cardiovascular.

El intentar refrenar los sentimientos de ira pero sin llegar realmente a controlarlos parece la variable más reveladora como factor de riesgo del padecimiento de trastornos cardiovasculares. Chida señala que el efecto dañino asociado a la ira y la 
hostilidad con los episodios de enfermedad cardiaca coronaria en las personas sanas era superior en los hombres que en las mujeres. "Esto sugiere que la acumulación de las respuestas ante el estrés en la vida diaria podría tener un mayor impacto en la enfermedad cardiaca en los hombres" (Chida, 2009: 940).

\section{Estrés psicológico}

Es un fenómeno complejo difícil de definir, medir y estudiar científicamente. Cuando las demandas impuestas por los eventos exceden la habilidad de una persona para enfrentarlos, se produce una respuesta psicológica de estrés compuesta por cogniciones y estados emocionales negativos. Algunos estudios apoyan la hipótesis de que el estrés psicológico puede jugar un rol en los eventos cardiacos, incluyendo la precipitación del infarto del miocardio. Lo que todavía no se sabe es qué tipo de estrés es más potente, o cómo la susceptibilidad individual, los perfiles conductuales preexistentes, o la respuesta a los estresores, afectan el proceso.

En mujeres se sabe que tienen un rol importante, fundamentalmente en desencadenar accidentes coronarios agudos, espasmos de la arteria coronaria y hasta miniinfartos cuando hay placas en las coronarias (Acevedo, Tagle y Simpfendorfer, 2001). Por otra parte, el estudio llevado a cabo por Elissa S. Epel (2006), realizado en la Universidad de Yale (EEUU), ha puesto de relieve que las mujeres más estresadas y a las que les resulta más difícil adaptarse a las situaciones estresantes tienen más probabilidades de acumular grasa en la cintura. La grasa abdominal en ambos sexos se ha asociado con un riesgo más elevado de enfermedades cardiovasculares

\section{Presión laboral}

Se ha encontrado que aquellos trabajos que suponen una gran demanda física o intelectual, excesiva responsabilidad o un alto grado de tensión, aumentan la probabilidad de que se presenten síntomas coronarios. Las mujeres se están incorporando cada vez más a este patrón de conducta: 
"Suelen ser más impacientes que hostiles, si bien se admite que la impaciencia deriva de la hostilidad. Lo cierto es que las adictas al trabajo superan con mucho a los hombres en cuanto al espectro de operaciones. Esto es, pueden ser tan autoritarias y competitivas como los hombres, pero son capaces de abarcar más actividades" (Sender, Salamero, Vallés y Diaz, 2001).

El grupo sueco de M. Frankenhaeuser en 1996 ha podido demostrar que la sobrecarga de trabajo de la mujer empleada, una baja capacidad para tomar decisiones y malas relaciones sociales constituyen los principales factores de riesgo para su salud.

\section{Agotamiento vital}

Un estado físico y emocional debilitante puede ser un precursor del infarto del miocardio. Appels (1989) sugirió el concepto de agotamiento vital inspirándose en los casos de excesiva fatiga y debilidad que suelen experimentar algunas personas antes de la ocurrencia del infarto de miocardio. Sus características son: la fatiga y pérdida de energía o vigor, aumento de irritabilidad, y sentimientos de desmoralización. Son también frecuentes los problemas del sueño.

Aislamiento social y falta de apoyo

El apoyo social o el grado en que uno está conectado con otros en la comunidad, ha surgido como un factor de riesgo inverso de considerable magnitud, no solamente para la enfermedad coronaria, sino para la morbilidad y mortalidad de todas causas. La influencia que ejerce el apoyo social en la relación existente entre los factores de riesgo y las enfermedades coronarias, ya sea debido al papel modulador que palia los efectos negativos del estrés, o ya sea debido al efecto directo que provoca sobre la percepción del objeto estresor en el sujeto. La mayor participación social de la mujer en roles laborales de mayor calidad, mejora su grado de satisfacción, disminuye su estrés psicológico y mejora su calidad de vida (Moen, 1989). 


\section{Depresión}

La depresión clínica se define por síntomas de sufrimiento emocional, disminución del interés en la vida, cambios en el apetito y patrones de sueño, dificultad para concentrarse, fatiga y agotamiento, disforia y en casos extremos, desesperanza, desamparo o desvalimiento e ideas suicidas. $\mathrm{Si}$, como han sugerido recientemente Schwartzman y Glaus (2000), los pacientes con depresión poseen un nivel elevado de riesgo para sufrir ECV, debería prestarse una especial atención a estas personas con objeto de prevenir posibles sucesos cardiovasculares futuros.

Existen además actualmente abundantes datos como para afirmar que la depresión constituye un factor de riesgo de primer orden en relación con posibles complicaciones o la muerte tras el infarto de miocardio (Óhman y Sundin, 1995; Burg y Abrams, 2001).

\section{Ansiedad}

La ansiedad puede aumentar el riesgo de enfermedad cardiovascular. Los hallazgos sugieren que la ansiedad fóbica puede aumentar el riesgo de enfermedad coronaria, por la variabilidad del ritmo cardiaco disminuido, un signo de un balance simpático vagal alterado, en la regulación autonómica del corazón. Trabajos publicados recientemente tienden a sugerir que la ansiedad no sólo está implicada en el desarrollo de problemas cardiovasculares sino también en el curso de la cardiopatía coronaria.

Algunos otros trabajos también han relacionado la ansiedad (tomada ésta en un sentido más genérico) con el curso de la cardiopatía coronaria. La morbilidad y mortalidad por eventos cardíacos se ha asociado a la presencia de trastornos de ansiedad (fobias y pánico). La ansiedad fóbica triplica el riesgo de cardiopatía coronaria fatal, siendo esta relación aún más alta cuando se trata de muerte coronaria súbita (Kawachi, Sparrow, Vokonas y Weiss, 1994). 


\section{Negación cardiaca}

La negación es una estrategia psicológica de afrontamiento que permite implicarse en una conducta con poca conciencia de las consecuencias. A veces, la negación es lo suficientemente poderosa como para permitir conductas negativas a pesar de ciertas alertas, por ejemplo en pacientes con enfermedades coronarias que continúan fumando. Como la negación de los eventos cardiacos puede llevar a una demora en la búsqueda de una consulta médica, las consecuencias pueden ser profundas. El individuo que retrasa la búsqueda de tratamiento aumenta el riesgo de daño del miocardio, morbilidad y mortalidad.

Algunos autores han sugerido que, más que la ansiedad, la hostilidad o la depresión por separado, es la combinación de las tres variables lo que realmente predispone hacia el desarrollo de problemas cardiovasculares. Esta idea ha sido descrita como un síndrome de distress (Denollet, 1997). Es decir, los pacientes con depresión y ansiedad, y/o los pacientes con depresión y hostilidad/irritabilidad, tendrían un riesgo superior a otros pacientes depresivos de desarrollar ECV.

\section{Manifestaciones diferenciales en mujeres y hombres}

La enfermedad cardiovascular femenina tiene unas características diferenciales. Predomina ligeramente la morbilidad cerebrovascular sobre la cardiopatía isquémica y las mujeres inician la enfermedad coronaria 10-15 años más tarde que el varón. Factores de protección diferentes: Los estrógenos, son un excelente factor de protección con un sistema de actuación multifactorial:

- Favorecen un correcto equilibrio en el perfil lipídico, disminuyendo las cifras de colesterol y triglicéridos.

- Protegen el endotelio vascular disminuyendo el riesgo de aterosclerosis.

- Diminuyen el tono vasoconstrictor vascular. 
- Disminuyen los niveles de glucemia basal.

Las mujeres presentan angina de pecho con más frecuencia y los hombres infarto agudo de miocardio, aunque ello no significa que ambos tipos de manifestaciones estén ausentes en el otro sexo. Los signos y síntomas del infarto agudo de miocardio en las mujeres pueden ser diferentes de los que presentan los hombres. Mientras que en el hombre el dolor acostumbra a ser en la parte anterior del tórax, y suele acompañarse de sudoración, las mujeres pueden en su lugar, sentir ardor en la región superior del abdomen o presentar dolor en el brazo izquierdo, espalda, mandíbula y cuello y pueden tener mareo, malestar estomacal y sudoración. Como pueden no sentir el típico dolor en la mitad izquierda del pecho, muchas mujeres podrían pasar por alto los síntomas que indican que están sufriendo un ataque al corazón.

En 1991 Ayanian y realizaron un estudio retrospectivo, en Estados Unidos, sobre unos 50000 pacientes de Massachusetts y unos 34000 de Maryland con el fin de determinar los procedimientos de control cuando llegaban al hospital con dolor torácico, la edad, los diagnósticos secundarios, la raza, y el estado de protección y seguridad social. Las tasas ajustadas por edad y sexo dieron unas referencias en los procedimientos efectuados que demostraron que las mujeres hospitalizadas tenían muchos menos procedimientos diagnósticos y terapéuticos que los hombres, observaron también que a igualdad de sintomatología cuando llegaban al hospital, los procedimientos, tanto terapéuticos iniciales como la trombólisis, el intento de deshacer los trombos, o después los procedimientos de investigación para conocer la extensión del problema, eran diferentes en los hombres que en las mujeres.

Estas diferencias tan claras en la utilización de procedimientos diagnósticos y terapéuticos en los servicios de urgencia y en la hospitalización de mujeres con problemas coronarios motivó que, en el año 1991, Bernardine Healy describiera el síndrome de Yentl en la prestigiosa revista The New England Journal of Medicine que determinó que las mujeres sufrían dolor anginoso antes de un infarto tan frecuentemente, incluso con efectos más debilitantes, como los hombres, pero que a las 
mujeres se les aplicaba únicamente una cateterización cardíaca (la mitad de las veces que a los hombres). En cambio, a una mujer que ya había tenido un infarto se le aplicaban las mismas técnicas de cataterización cardíaca o de revascularización (Valls Llobet, 2003).

Además de las diferencias en exploración y diagnóstico, se han puesto de manifiesto diferencias en el tratamiento a saber:

- Efectos secundarios de los antihipertensivos según el sexo, no estudiados.

- Aspirina: un medicamento muy eficaz para la prevención primaria de infartos en los hombres, pero no parece serlo tanto en las mujeres. Lo contrario es cierto para la prevención de la enfermedad cerebrovascular o ictus.

- Farmacocinética: estudiada aunque no conocida de los fármacos antiarrítmicos, como la conterfenadina, que producen más mortalidad en mujeres (debido a que las mujeres tienen una bomba de potasio diferente en sus células cardíacas).

- Fármacos hipolipemiantes: no estudiados mayoritariamente en el sexo femenino, producen miopatías como efecto secundario sobre todo entre mujeres con hipotiroidismo. Según una revisión de metaanálisis de 13 ensayos (Pignone et al., 2003) en las mujeres sin enfermedad isquémica previa, los fármacos hipolipemiantes (estatinas) no parecen contribuir a disminuir la mortalidad total o la coronaria, aunque existen indicios de que éstos fármacos podrán disminuir los episodios vasculares no mortales

Cuando, en 1991, Bernardine Healy analizó la mala praxis de los servicios de cardiología americanos, observó de dónde nacía este posible sesgo inconsciente y se remontó a los años ochenta, en que todos los programas de investigación que se realizaban relacionados con las enfermedades cardiovasculares estaban dirigidos al estudio del tabaquismo, los lípidos, las hiperlipemias o de los tratamientos y prevención 
de algunos fármacos en la enfermedad cardiovascular; todas estas investigaciones se habían hecho sólo en hombres dentro de la cohorte del estudio.

Por tanto, el primer dato es que las mujeres durante años fueron excluidas sistemáticamente de los ensayos clínicos. Muchos ensayos clínicos se habían realizado sólo entre hombres, suponiendo que los resultados se podían aplicar automáticamente a las mujeres, o que las mujeres estaban naturalmente protegidas de las enfermedades cardiovasculares y que, en cambio, únicamente los hombres corrían unos determinados riesgos.

Los factores de riesgo para la muerte súbita derivados del Framingham Heart Study (1987) llevado a cabo en 1948, después de 26 años de seguimiento son diferentes para hombres y mujeres. Para los hombres, los factores de riesgo más importantes de la enfermedad cardiovascular son, por este orden: la edad, el colesterol sérico, el número de cigarrillos fumados a diario, el peso relativo y la tensión arterial sistólica. En cambio, para las mujeres los tres principales predictores de enfermedad cardiovascular son: la edad, la capacidad vital (la capacidad respiratoria máxima) y el hematocrito, que indica el grado de anemia o el volumen de glóbulos rojos y, como dato al margen, en mujeres de más de 60 años, el colesterol y la glucosa también son otros factores de riesgo (Schatzkin, 1984).

Un ejemplo paradigmático de la invisibilidad en el estudio de las diferencias, creyendo que las mujeres están protegidas por su propia biología, ha sido precisamente la terapia hormonal sustitutiva aplicada a las mujeres después de la menopausia y la enfermedad cardiovascular. Esta relación se estableció sin ensayos clínicos de larga duración, tan sólo con ensayos de tres o cuatro meses viendo si mejoraba la calidad de vida inmediata, la sequedad vaginal y los sofocos y, en cambio, no se estudió el efecto a largo plazo sobre la enfermedad cardiovascular.

Con esta creencia en mente, se decidió afirmar que la terapia hormonal sustitutiva en la menopausia protegería a las mujeres de la enfermedad cardiovascular; por suerte, en este caso se aplicó la medicina basada en la evidencia, que quiere decir la medicina basada en los ensayos clínicos, sobre todo los incentivados y realizados desde 
las entidades no lucrativas o desde la salud pública de cada país. Se observó un mayor riesgo de enfermedad coronaria, independientemente de la edad, de cáncer de mama, de ictus y de trombosis venosa/tromboembolia, con una reducción significativa de la fractura de cadera y una discreta reducción del cáncer colorrectal. De hecho, se demostró que había un considerable mayor número de muertes por trombosis durante los primeros años del estudio entre el grupo tratado con terapia hormonal sustitutiva que en el grupo placebo (Hulley et al., 1998 y Rossouw et al., 2002).

Todos estos aspectos podemos estructurarlos de un modo resumido al considerar que en las enfermedades cardiovasculares, especialmente en las de origen ateroesclerótico cabe distinguir aspectos relacionados con el sexo y con el género. Entre los principales aspectos diferenciales relacionados con el sexo cabría destacar:

- Diferencias en la anatomía y fisiologías cardiocirculatorias y cerebrales, entre las que se encuentran la presencia de los receptores de estrógenos, que modulan de distinta forma las respuestas fisiológicas y el menor calibre y fragilidad de las arterias coronarias, que puede jugar un papel adverso en el pronóstico de la cardiopatía isquémica.

- La presentación de las manifestaciones clínicas de las enfermedades ateroescleróticas a edades más avanzadas en las mujeres debido al efecto protector de las hormonas femeninas o estrógenos sobre el metabolismo de los lípidos, de la presión arterial, y la fisiología del endotelio.

- La mayor prevalencia de sobrepeso, obesidad e hipertensión a partir de los 50 años.

- Las dificultades de diagnóstico de la cardiopatía isquémica con las mismas pruebas convencionales utilizadas en el hombre como por ejemplo la prueba de esfuerzo.

- Las diferentes formas de presentación clínica.

- Diferencias en el metabolismo y efecto de determinados fármacos, ocasionando así mismo diferencias en la supervivencia y pronóstico. 
- Mayor frecuencia de comorbilidades como la diabetes y la hipertensión arterial que confieren un peor pronóstico.

Entre los principales aspectos diferenciales relacionados con el género cabe destacar:

- El relativo conocimiento de la cardiopatía isquémica como un problema de salud que también afecta a la mujer.

- Diferencias en la percepción personal, social y sanitaria de los síntomas.

- Infradiagnóstico de la cardiopatía isquémica en la mujer.

- Demora en recibir atención sanitaria adecuada en presencia de un episodio cardiovascular agudo.

- Menor aplicación de tratamientos eficaces e intensivos.

- Menor evidencia científica de la eficacia de determinados fármacos y otras intervenciones.

- Distintos factores psicosociales que influencian el perfil de los factores de riesgo en la mujer.

\section{Conclusiones}

Tanto para hombres como para mujeres podríamos establecer unos objetivos principales de prevención en pacientes con riesgo de enfermedad cardiovascular, o con enfermedad cardiovascular establecida con el fin de prevenir la invalidez y las muertes prematuras, reducir la incidencia y la recurrencia de eventos cardiovasculares clínicos de infarto agudo de miocardio, ictus y enfermedad arterial periférica, si consideramos que la ocurrencia epidémica de las ECV está estrechamente asociada con hábitos de vida y factores fisiológicos modificables. 
La modificación de los factores de riesgo ha demostrado de forma inequívoca que reduce la mortalidad y la morbilidad, especialmente en personas con ECV diagnosticada o no. Esos objetivos estarán encaminados a:

- Minimizar o eliminar tabaco y alcohol.

- Mantener una alimentación cardiosaludable.

- $\quad$ Ser activo físicamente.

- Evitar situaciones de stress que pueden generar trastornos como depresión, ansiedad, etc.

- Chequeo periódico en el que se controlaran los siguientes parámetros :

- $\quad$ Índice de masa corporal $<25 \mathrm{~kg} / \mathrm{m} 2$

- $\quad$ Presión arterial $<140 / 90 \mathrm{mmHg}$

- Colesterol total $<200 \mathrm{mg} / \mathrm{dl}(5,2 \mathrm{mmol} / \mathrm{l})$ en general,

- Colesterol-LDL < $130 \mathrm{mg} / \mathrm{dl}$ (3,4 mmol/l) en general,

- Glucemias bien controlado en todos los diabéticos/as

Para conseguir cambios intensos de estilo de vida en pacientes con enfermedad cardiovascular y en pacientes de riesgo es necesario involucrar directamente al paciente o a la persona de alto riesgo en el cambio del estilo de vida. Podemos establecer algunas estrategias para el consejo conductual más efectivo:

- Desarrollar un compromiso terapéutico con el/ la paciente.

- Asegurarse de que el/ la paciente comprende la relación entre conducta, salud y enfermedad.

- Involucrar a los/ las pacientes en la identificación y selección de los factores de riesgo que deben cambiar.

- Ayudar al paciente a entender las dificultades para el cambio de conducta, y conseguir el compromiso para el cambio. 
- Usar una combinación de estrategias, incluyendo el refuerzo de la capacidad propia de los/ las pacientes para cambiar, y diseñar un plan de modificación de estilos de vida.

- Hacer un seguimiento del progreso mediante visitas de control.

- Implicar a otros profesionales sanitarios y sociales siempre que sea posible.

Y específicamente en cuanto a las mujeres... Es ineludible corregir los posibles sesgos de género: en la investigación, en la prevención, en la atención, en la formación de las y los profesionales de la salud, en la información a las propias mujeres, en la producción del conocimiento incluyendo a mujeres y hombres en las investigaciones, corregir el posible infradiagnóstico en las mujeres, corregir los posibles diagnósticos erróneos y mejorar el conocimiento epidemiológico y clínico. Es necesario descubrir los mecanismos que perpetúan esta situación en los y las profesionales y en las pacientes, que inducen a inequidades ( injustas desigualdades) entre hombres y mujeres en el autocuidado y en el acceso a los servicios y en los resultados de salud, que hay que corregir para que nuestros programas sean más efectivos.

Es obligado reconocer que las mejoras en salud necesitan un abordaje más allá del hospital, en la atención primaria de salud, en la Salud Pública y en la misma sociedad y desde luego en las propias mujeres, las cuales han de conocer que esta enfermedad les afecta, y han de saber reconocer su comienzo, su prevención y su rehabilitación... y saber buscar ayuda a tiempo, ya que permanecer sin acudir al sistema sanitario podría costarles la vida. 


\section{BIBLIOGRAFÍA}

- Acevedo M.; Tagle R. y Simpfendorfer C. (2001): "Non-traditional risk factors for atherosclerosis". En Rev Med Chile, oct., vol.129, n’. 10, pp. 234-239.

- Alegría E, et al. (1991) “Cardiopatía isquémica en la mujer.” En Revista Española Cardiología, $\mathrm{n}^{\circ} 44$.

- Appels, A. (1989): "Loss of control, vitalexhaustion, and coronary heart disease". En A. Steptoe y A. Apples (eds.): Stress, personal control and Elath. New York, pp. 215235.

- ASOCIMED guías clínicas (2001): "Pautas diagnóstico - terapéuticas para la práctica clínica .Tema, condición o patología: Dislipidemias”, [en línea]. Disponible en: http://www.asocimed.cl/Guias\%20Clinicas/endocrinologia/dislipidemias.html 10/01/2010].

- Ayanian, J. Z. y Epstein, A. M. (1991): "Differences in the use of procedures between women and men hospitalized for coronary heart disease". En The New England Journal of Medicine, Jul, $\mathrm{n}^{\mathrm{o}}$. 25, vol. 325(4), pp. 221-225.

- Banegas J. et al. (2006): "Estudio PREVENCAT". En Revista Española de Cardiología, no. 6, p. 3-12.

- Burg, M. M. y Abrams, D. (2001): "Depression in chronic medical illness: The case of coronary heart disease". En Journal of Clinical Psychology, vol. 57, n. 11, pp. 13231337.

- Castro Beiras, A. (1999): Enfermedad cardiovascular en la mujer. Barcelona: Masson.

- CIE - X Versión (2007) - "Versión X de la Clasificación Internacional de Enfermedades de la OMS", [en línea]. Disponible en: http://apps.who.int/classifications/apps/icd/icd10online/ [10/01/2010].

- Collins P. et al., (2007) "El control del riesgo cardiovascular es esencial en mujeres perimenopáusicas”. En European Heart Journal, vol. 28, n. 16, pp: 2028-20402.

- Conroy R. M., et al. (2003): "Estimation of ten-year risk of fatal cardiovasculardisease in Europe". En European Heart Journal, vol. 24, n. 11, pp. 987-1003.

- Chida, Y. y Steptoe, A. (2009): "The association of anger and hostility with future coronary heart disease: A meta-analytic review of prospective evidence". En Journal of 
the American College of Cardiology, vol. 53, $\mathrm{n}^{\circ} .11$, pp. 936-946, [en línea] Disponible en: http://www.ucl.ac.uk/psychobiology [Acceso 30/02/2010].

- Denia Ruiz, F. y Mingote Adán, C. (2000): "Factores psicosociales que influyen en el desarrollo de la enfermedad coronaria (EC) en la mujer". En II Jornadas de Salud Mental y Género. Madrid: Ed. Instituto de la Mujer (Ministerio de Igualdad), pp 77- 91. - Denollet, J. (1997): "Personality, emotional distress and coronary heart disease". En European Journal of Personality, vol. 11, nº. 5, pp. 343-357.

- Eckel R. H.; Grundy S. M. y Zimmet P. Z. (2005): “The metabolic syndrome”. En Lancet. 2005; vol. 365, pp. 1415-28.

- Epel E.; Jue L. y Wilhem F. (2006) "Envejecimiento celular en relación con el stress y factores de riesgo de enfermedad cardiovascular". En Psychoneuroendocrinology, vol. 31, pp 277-287.

- Framingham Heart Study (1987): "30 year follow-up”. Section 34. En Kannel W. B.; Wolf P. A. y Garrison R. J. (eds.): The Framingham Study: an Epidemiological Investigation of Cardiovascular disease. Bethesda: National Institutes of Health, $\mathrm{n}^{\mathrm{o}} 87$, p. 2703.

- Frankenhaeuser M. (1996): "Sterss and gender". En European Revieuw vol. 4. no 4, pp. 313-327.

- Fiol M. et al. (2001): "Variabilidad en el manejo hospitalario del infarto agudo de miocardio en España. Estudio IBERICA”. En Revista Española Cardiología l., nº. 54, pp. 443-452.

- García de Vicuña, B. et al. (2003): Características mínimas de un programa de rehabilitación cardiaca en la Comunidad Autónoma del País Vasco. Vitoria-Gasteiz: Departamento de Sanidad, Gobierno Vasco.

- Garfinkel A, et al. 1992). "Controlling cardiac chaos”. En Science, vol. 1557, n’. 5074, pp 1230-1235.

- Gil M. et al. (1999) "Relationship of therapeutic improvements and 28-day case fatality in patients hospitalized with acute myocardial infarction between 1978 and 1993 in the REGICOR study, Gerona, Spain. The REGICOR Investigators”. En Circulation, april, vol. 99, no. 13, pp.1767-73. 
Haffner S. M. et al. (2006): "Waist circumference and body mass index are both independently associated with cardiovascular disease. The international Day for the Evaluation of Abdominal Obesity (IDEA) survey". En: Journal of the American College of Cardiology, February, vol. 47, nº 4, supl. A, pp. 842-846.

- Healy, B. (1991): “The Yentl syndrome". En The New england journal of Medicine, Jul 24; vol. 325, nº 4, pp 221-225.

- Hulley S. et al. (1998): "Randomized trial of estrogen plus progestin for secondary prevention of coronary heart disease in postmenopausal women. Heart and Estrogen/progestin Replacement Study (HERS) Research group”. En The Journal of the American Medical Association, august, vol. 280, nº 7, pp. 605-613.

- Instituto Nacional de Estadística: "Defunciones según la causa de muerte”, [en línea] Disponible en: http://www.ine.es [21/ 12 / 209].

- Kahn E. et al. (2002) "The effectiveness of interventions to increase physical activity. A systematic review". En American Journal of Preventive Medicine, may, vol. 22, nº . 4, pp. 73-107.

- Kawachi, I.; Sparrow, D.; Vokonas, P. S. y Weiss, S. T. (1994): "Symptoms of anxiety and risk of coronary heart disease: The Normative Aging Study". En Circulation, vol. 90, pp. 2225-29.

- Lusiardo, M. (2005): "Mente y Corazón. Factores psicosociales de riesgo cardiovascular". En Revista Salud Militar, vol. 27, n. 1, pp. 75 - 82.

- Friedman M. y Rosenman, R. H. (1974): “Type A Behavior and Your Heart”, [en línea] Disponible en http://www.stress.org/topic-heart.htm [16 / 01 / 2010].

- Moen P. (2005): "Las desigualdades de género en salud: selección estratégica". En Journal Gerontologi Society Science B Psychology Science. n. 2, pp. 99-108.

- Muñiz García J. et al. (2006): "Insuficiencia cardíaca en España. Epidemiología e importancia del grado de adecuación a las guías de práctica clínica". En Revista Española de Cardioliología, $\mathrm{n}^{\circ}$ 6, pp. $6-8$.

- Muñiz García, J. y Castro Beiras, A. (1999): "Las enfermedades cardiovasculares en España”. En Cuenta y Razón, $\mathrm{n}^{\circ}$. 113, [en línea] Disponible en: http://www.cuentayrazon.org/revista/pdf/113/Num113_006.pdf [10 / 01/2010].

- Óhman, A. y Sundin, O. (1995). "Emotional factors in cardiovascular disorder". En Current Opinión in Psychiatry, vol. ${ }^{\circ}$ 8, nº. 6, pp. 410-413. 
- Protege y restablece: “Accidentes vasculares del sistema nervioso central”, [en línea]. Disponible en: http://www.protegeyrestablece.cl/Accidentes\%20Vasculares\%202.htm [30/02/2010].

- Petersen, S. et al (2005): European cardiovascular disease statistics. Oxford: British Heart Foundation.

- Pignone, M.; Phillips, C. y Mulrow, C. (2000): "Use of lipid lowering digs for primary prevention of coronary heart disease: meta-analysis of randomised trials". En British Medical Journal, vol. 321, nº 7267, pp. 983-6.

- Rodríguez Artejo, F.; Villar Álvarez, F. y Banegas Banegas, R. (2000): "Epidemiología de las enfermedades cardiovasculares y de sus factores de riesgo en España”. En Medicine 2000; nº. 8, pp. 12-18.

- Rodríguez Artalejo F. et al. (2000): "Variación geográfica en las hospitalizaciones y la mortalidad por insuficiencia cardiaca congestiva en España, 1980-1993”. En Revista Española de Cardiología, $\mathrm{n}^{\circ}$. 53, pp.776-782.

- Rolla A. (2003): "Nuevos Componentes del Síndrome de Resistencia Insulínica” [en línea]. Disponible en http://www.mednet.cl/link.cgi/Medwave/Congresos/Nutricion/1/999 [12 / 01/ 2010].

- Rossouw, J. E. et al. (2002): "Risks and benefits of estrogen plus progestin in healthy postmenopausal women: principal results from the Women's Health Initiative randomized controlled trial". En Journal of the American Medical Association, jul, vol. 288, no. 3, pp 321-323.

- Sandin, B. (2002): "Papel de las emociones negativas en el trastorno Cardiovascular: un análisis crítico". En Revista de Psicopatología y Psicología Clínica, vol. 7, nº 1, pp. 1-18.

- Sans S. et al. (2001): "Tendencias de la prevalencia, conocimiento, tratamiento y control de la hipertensión arterial entre 1986 y 1996: estudio MONICA-Cataluña”. En Medicina Clinica; vol. 117, no. 7, pp. 246-53.

- Schatzkin, A. et al. (1984): “The epidemiology of sudden unexpected death: Risk factors for men and women in the Framingham Heart Study". En American Heart Journal, june, vol.107, no.6, pp 1300-1306. 
- Schwartzman, J. B. y Glaus, K.. D. (2000): "Depression and coronary heart disease in women: Implications for clinical practice and research". En Professional PsychologyResearch and Practice, vol. 3, nº 1 , pp 48-57.

- Sender R. et al. (2001): "Variables psicológicas predictoras de psicopatología". En Educación Médica, $\mathrm{n}^{\circ}$. 4: p. 119.

- SEMERGEN (2005): "Guía sobre Hipertensión arterial. Sociedad Española de hipertensión - Liga española para la lucha contra la Hipertensión”, [en línea] Disponible en http://www.seh-lelha.org/guiahta05.htm [10/01/2010].

- Stuardo, W. F. y Navarro, R. J. (2003): "Relación entre la ingestión de alcohol y desarrollo de cardiopatía isquémica". En Revista Mexicana de Cardiología, vol. 14, $\mathrm{n}^{\mathrm{o}} .4$, pp. 134-137.

- Sans Menéndez, S. (2006): "Institut d' Estudis de la Salut”. Barcelona. Programa de Formación de Formadores/as en Perspectiva de Género en Salud. Ministerio de Sanidad y Consumo. Universidad Complutense de Madrid.

- Sans Menéndez, S. (2006): “Enfermedades Cardiovasculares”. Módulo 6 del Programa de Formación de Formadores/as en Perspectiva de Género en Salud. Ministerio de Sanidad y Consumo. Universidad Complutense de Madrid, [en línea] Disponible en: http://www.msc.es/organizacion/sns/planCalidadSNS/pdf/equidad/07modulo_06.pdf [10/01/2010].

-Vaccarino, M. D. et al. (2009): “Sex Differences in Mortality After Acute Myocardial Infarction”. En Archives International Medicine, vol. 169, nº. 19, pp. 1767-1774.

- Vessey M.; Painter R. y Yeates D. (2003): "Mortalidad Relacionada con el Uso de Anticonceptivos Orales y el Consumo de Cigarrillos”. En The Lancet, nº. 362: pp. 185191

- Valls Llobet, C. (2003): “Aspectos biológicos y clínicos de las diferencias". En Revista Quark, enero-marzo, nº. 27, Barcelona, pp: 41-53.

- Villar Álvarez, F. y Banegas Banegas, J. R. (2003): "Las enfermedades cardiovasculares y sus factores de riesgo en España: hechos y cifras". En Informe SEA. Madrid: Ergon, pp. 112-124.

- Williams, R. B. (1987): "Refining the Type A hypothesis: emergence of the hostility complex". En: American Journal of Cardiology, n. 60, pp.27-32. 